\title{
The Mechanism of Action of Xanthine Oxidase
}

\section{THE RELATIONSHIP BETWEEN THE RAPID AND VERY RAPID MOLYBDENUM ELECTRON-PARAMAGNETIC-RESONANCE SIGNALS}

\author{
By ROBERT C. BRAY,* STEVEN GUTTERIDGE,* DAVID A. STOTTER $\ddagger$ \\ and STEPHEN J. TANNER* \\ *School of Molecular Sciences, University of Sussex, Falmer, Brighton BN1 9QJ, Sussex, U.K., and \\ $\dagger$ Department of Chemistry, University of Essex, Wivenhoe Park, Colchester CO4 3SG, Essex, U.K.
}

(Received 19 September 1978)

\begin{abstract}
On the basis of the work of Gutteridge, Tanner \& Bray [Biochem. J. (1978) 175, 887-897] and of other data in the literature, a mechanism for the reaction of xanthine oxidase with reducing substrates is proposed. In the Michaelis complex, xanthine is bound to molybdenum via the N-9 nitrogen atom. Coupled transfer of two electrons to molybdenum and the $\mathrm{C}-8$ proton to the enzyme yields (Enzyme)-Mo-SH. Concerted with this process, reaction of the xanthine residue with a nucleophile in the active centre yields a covalent intermediate that breaks down to give the product by alternative pathways at high and at low $\mathrm{pH}$ values.
\end{abstract}

Olson et al. (1974) put forward a comprehensive mechanism explaining the processes of reduction and reoxidation of the enzyme xanthine oxidase by its substrates, xanthine and oxygen. The main points, involving the binding and reaction at the molybdenum centre of the enzyme of up to three successive xanthine molecules, have been widely accepted (see Bray, 1975). Reaction of the xanthine molecules was taken to involve a covalently bound intermediate, with transfer of two electrons from the substrate to the enzyme. These electrons were initially accepted by molybdenum, which was thereby reduced from the six- to the four-valent state, but were then rapidly redistributed among the other reducible centres. It was suggested that the xanthine residue, having lost the hydrogen from its $\mathrm{C}-8$ position, was bound via this carbon atom to the terminal sulphur atom of a persulphide group located near to molybdenum in the active centre. Hydrolysis of the intermediate yielded the product, uric acid, and the free (reduced) enzyme.

Almost all information about molybdenum in xanthine oxidase has been derived from e.p.r. spectroscopy. According to Olson et al. (1974), the e.p.r. properties of the intermediate species were supposed to depend on the number of electrons that the enzyme half-molecule had accepted, as well as on the $\mathrm{pH}$ of the solution. Thus at high $\mathrm{pH}$ values, in the reaction of the first xanthine molecule with the oxidized enzyme, the intermediate gave the Very

‡ Present address: Shell Toxicology Laboratory (Tunstall), Sittingbourne Research Centre, Sittingbourne, Kent ME9 8AG, U.K.
Rapid molybdenum(V) e.p.r. signal, but under all other conditions it gave the Rapid signal.

More recently, Gutteridge et al.(1978a) emphasized the role of a proton-accepting group in the enzyme active centre in abstracting the hydrogen from the substrate C-8 position. Further, Gutteridge et al. $(1978 b)$ presented evidence that this accepting group is (Enzyme)-Mo=S, transformed on protonation to (Enzyme)-Mo-SH. They concluded that the sulphur atom of the accepting group is the one liberated as thiocyanate during inactivation of the enzyme by cyanide. This is the sulphur originally presumed by Massey \& Edmondson (1970) to be present as a persulphide participating in the catalytic reaction. Finally, Tsopanakis et al. (1978) carried out pHjump studies at subzero temperatures on the Very Rapid signal, generated on treating the enzyme with xanthine in aq. dimethyl sulphoxide. Results did not support the claim of Edmondson et al. (1973) and Olson et al. (1974) that the Rapid and Very Rapid signals are in $\mathrm{pH}$-dependent equilibrium with one another.

Partial revision of the reaction mechanism of Olson et al. (1974) thus becomes necessary. In the present paper we propose a new mechanism for interaction of the enzyme with reducing substrates.

\section{Kinetic Considerations and the Development of a Reaction Scheme}

An acceptable reaction mechanism for xanthine oxidase must account for the kinetic relationships 
between the Very Rapid and the Rapid Mo(V) e.p.r. signals. Olson et al. (1974) assumed that they were related as shown in Scheme 1. This accounts for the transient nature of the Very Rapid signal, but is not compatible with pH-jump studies (Tsopanakis et al., 1978), nor is it readily reconciled with the direct hydrogen-transfer data of Gutteridge et al. (1978a).

Any mechanism must involve fission of the carbonhydrogen bond at the C-8 position of the xanthine molecule. This might be accompanied by transient attachment to the enzyme of one, or perhaps of both, of the two parts of the substrate molecule, that is the proton and the deprotonated xanthine residue. In fact there is good evidence for attachment to the enzyme of each portion of the xanthine molecule separately, but not both of them together. Transfer to the enzyme of the C-8 proton was studied by Gutteridge et al. $(1978 a)$. The proton is detected as the enzyme-bound proton interacting with Mo in the Rapid signal, as this signal is initially formed, particularly when 1 -methyl $\left[8-{ }^{2} \mathrm{H}\right]$ xanthine is used as substrate. This proton, however, subsequently exchanges quite fast with protons of the medium. Concerning the larger part of the substrate molecule, there seems no reason to dispute the suggestion of Olson et al. (1974) that this becomes covalently bound to the enzyme, yielding the Very Rapid signal. In keeping

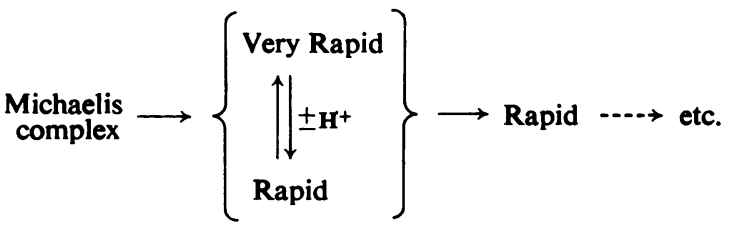

Scheme 1. Relationship between the Rapid and Very Rapid $M o(V)$ e.p.r. signals, as suggested by Olson et al. (1974) with this, Tanner et al. (1978) have recently observed a splitting, though a rather small one, of the Very Rapid signal by ${ }^{13} \mathrm{C}$ incorporated specifically into the $\mathrm{C}-8$ position of the xanthine molecule.

It is important to emphasize that, in the signalgiving species, the binding of the two portions of the substrate molecule to the enzyme seems mutually exclusive. Thus there are no protons, derived from the substrate or otherwise, interacting with Mo in the Very Rapid signal. Similarly in the Rapid signal, the evidence (Gutteridge et al., 1978a) seems to exclude any specific binding of a substrate residue in the signal-giving species.

All these findings may be rationalized and incorporated into a single catalytic mechanism (Scheme 2), by postulating a common precursor of the Very Rapid and Rapid signal, which is, however (for reasons discussed below) not detected by e.p.r. during substrate turnover. The invisible species, bearing both xanthine moieties, breaks down by alternative routes at high and at low $\mathrm{pH}$ values. Allowance is made for separate Rapid species with and without the proton transferred from the substrate (Gutteridge et al., 1978a).

By using Scheme 1, Olson et al. (1974) obtained acceptable computer simulations of the kinetics of reduction of xanthine oxidase by xanthine, at high or low concentrations, at $\mathrm{pH} 8.5$. They suggested that variations in the $\mathrm{p} K$ for the Very Rapid-Rapid interconversion would account for lower (or vanishingly small) amounts of Very Rapid signal seen when other substrates were used. Scheme 2 should be equally capable of accounting for the observed kinetics, and we note, qualitatively, that speeding up of the pathway via (IV) as the $\mathrm{pH}$ value decreases should diminish the amount of Very Rapid signal, without, however, changing the time for maximum yield of this signal. This is what

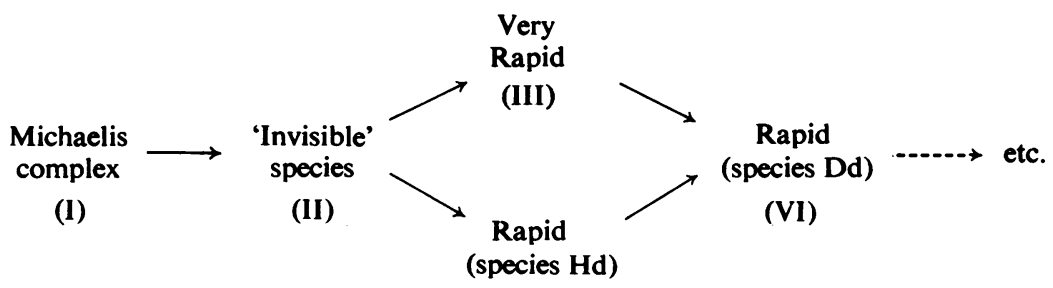

(IV)

Scheme 2. Relationship between the Rapid and Very Rapid Mo(V) e.p.r. signals (present work), accounting for direct transfer of hydrogen from the substrate to the enzyme

The pathway via (III) is favoured at high $\mathrm{pH}$ and that via (IV) at low $\mathrm{pH}$. The nomenclature of the Rapid species is that of Gutteridge et al. (1978a); for species Hd the proton giving the main splitting has been transferred from the substrate, whereas the one giving the smaller splitting is from the medium; for species Dd, both protons are from the medium. The numbers of the various species correspond to those shown in Scheme 3. 


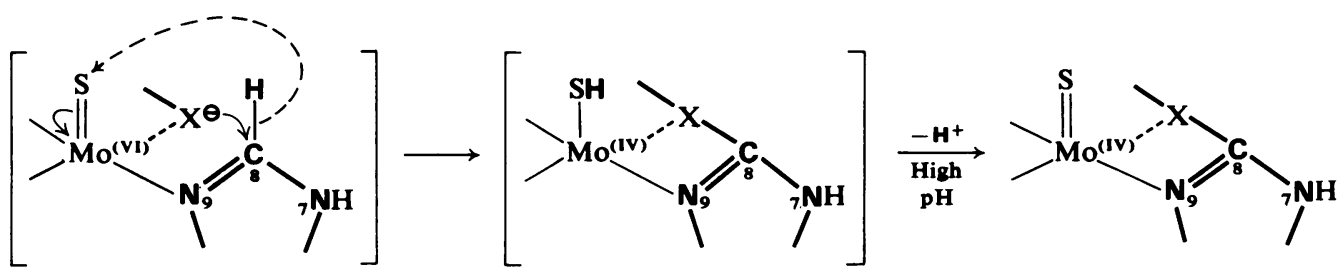

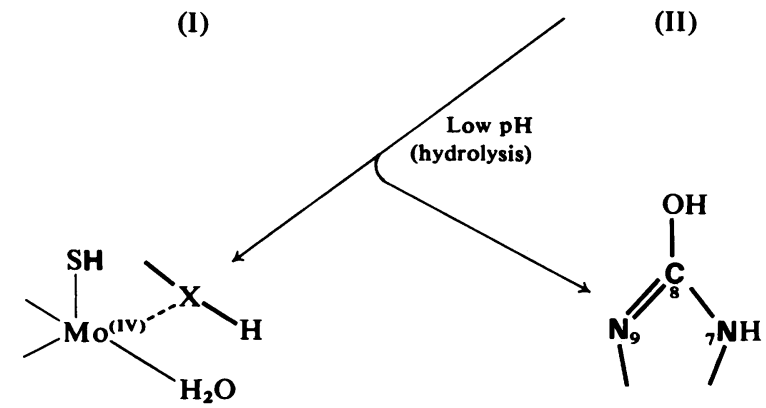

(IV)
(V)

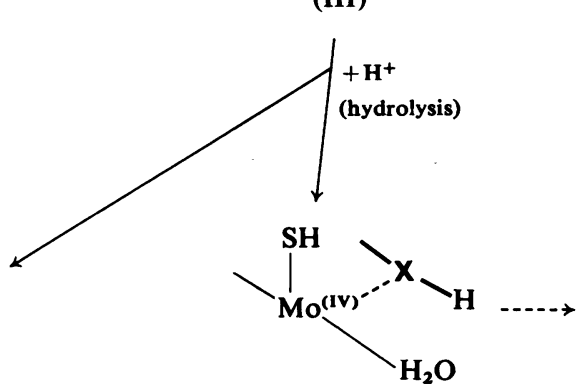

(VI)

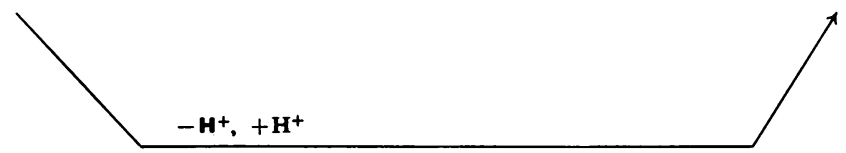

Scheme 3. Proposed chemical nature of the various signal-giving species in reduction of xanthine oxidase by xanthine The numbering of the species corresponds to that in Scheme 2. Bold-face type denotes atoms of, or derived from, the 7-, 8- and 9-positions of the substrate molecule. The Michaelis complex (I) and the intermediate (II) (shown in square brackets) are not detected by e.p.r.; (III) gives the Very Rapid signal and (IV) and (VI) the Rapid; (V) is the product, uric acid. In the signal-giving species, though $\mathrm{Mo}^{(\mathrm{IV})}$ is shown, internal oxidation-reduction equilibria with iron-sulphur and flavin will yield some $\mathrm{Mo}^{(\mathrm{V})}$. In the Rapid species, the strongly coupled proton is the one attached to $\mathrm{S}$ and the weakly coupled one that on X; the aquo complex is depicted, although complexes with product, substrate or buffer constituents are also possible. The geometry of the ligands, the nature of the nucleophile, $X$, and whether other atoms intervene between it and Mo are uncertain; electron transfer is represented schematically.

Edmondson et al. (1973) observed experimentally with xanthine as substrate when varying the $\mathrm{pH}$ value.

\section{Chemical Interpretation}

A chemical interpretation of Scheme 2, is shown in Scheme 3, taking account of (Enzyme)-Mo=S as the proton-accepting group in the enzyme (Gutteridge et al., 1978b). Attack on the substrate takes place in the Michaelis complex (I), in which the substrate is co-ordinated, via its N-9 position, to the anionbinding site in the enzyme (Gutteridge et al., 1978a; Bergmann \& Levene, 1976). The nucleophile, X, rather than the 'persulphide' group of Massey \& Edmondson (1970), is depicted as an undefined species attacking the C-8 carbon of the xanthine. The reaction is, overall, one of coupled transfer of two electrons to molybdenum (probably via the N-9 atom) and of the proton to the acceptor group (cf. Stiefel, 1973), while the xanthine C-8 carbonium ion reacts with the nucleophile, $X$.

At low $\mathrm{pH}$, after this initial reaction, hydrolysis of (II) to yield the product (V) and the Rapid species (IV) takes place before the proton transferred to the enzyme has exchanged into the solvent. At high $\mathrm{pH}$, on the other hand, deprotonation to yield the Very Rapid species (III) precedes hydrolysis and product liberation. We are not certain why the intermediate (II) has not been detected by e.p.r. Possibly its existence is so transient that it has so far been missed. Alternatively, its internal oxidationreduction equilibrium may be such that $\operatorname{Mo}(\mathrm{V})$ is not favoured.

Geometry of the ligands around Mo, the nature of the nucleophile, $X$, and whether or not other atoms intervene between it and the Mo atom are not certain. However, noting that both the strongly coupled and the weakly coupled proton are present 
together in the Rapid signal and that neither is present in the Very Rapid signal, we have assumed, in Scheme 3, that X, when it is not substituted by the xanthine residue, bears the weakly-coupled proton of the Rapid signal.

\section{Further Consideration}

A further signal derived from functional enzyme (Edmondson et al., 1972) is Inhibited (Pick et al., 1971; Tanner \& Bray, 1978b). This is believed to correspond to an RCO group bound in the active centre, where $\mathrm{RCHO}$ or $\mathrm{RCH}_{2} \mathrm{OH}$ is the aldehyde or alcohol used to inactivate the enzyme and generate the signal. We now suggest that Inhibited represents the 'wrong-way-round' reaction of a substrate-type molecule at the active centre, with the sulphur atom now acting as a nucleophile rather than as a proton acceptor. Thus, in Inhibited, we would have (Enzyme) -Mo-S-COR. Similarly, since Resting II (Lowe et al., 1976) is regarded (Tanner \& Bray, 1978a) as the desulpho enzyme analogue of Inhibited, we propose that in Resting II we have (Enzyme)-Mo-O-COR.

Scheme 3 suggests further studies. Under some conditions the 'invisible' species (II) might become detectable. Also, particular acylating or alkylating agents might give rise to Very Rapid species or to their as yet unknown desulpho analogues. Further work is also required on direct oxygen transfer in xanthine oxidase-catalysed reactions. Murray et al. (1966) claimed a small but significant transfer of ${ }^{18} \mathrm{O}$ from ${ }^{18} \mathrm{O}_{2}$ to uric acid during xanthine oxidase turnover at pH8.9. We suggest, if direct oxygen transfer is substantiated, that it could only occur via an oxygenated enzyme species, this probably being of the type (Enzyme)-Mo=O.
We thank Professor F. Bergmann for valuable discussions. The work was supported by a Programme Grant from the Medical Research Council. S. J. T. was in receipt of a Studentship from the Science Research Council.

\section{References}

Bergmann, F. \& Levene, L. (1976) Biochim. Biophys. Acta 429, 672-688

Bray, R. C. (1975) Enzymes 3rd Ed. 12, 299-419

Edmondson, D., Massey, V., Palmer, G., Beacham, L. M. \& Elion, G. B. (1972) J. Biol. Chem. 247, 1597-1604

Edmondson, D. E., Ballou, D., Van Heuvelen, A., Palmer, G. \& Massey, V. (1973) J. Biol. Chem. 248, 6135-6144

Gutteridge, S., Tanner, S. J. \& Bray, R. C. (1978a) Biochem. J. 175, 869-878

Gutteridge, S., Tanner, S. J. \& Bray, R. C. (1978b) Biochem. J. 175, 887-897

Lowe, D. J., Barber, M. J., Pawlik, R. \& Bray, R. C. (1976) Biochem. J. 155, 81-85

Massey, V. \& Edmondson, D. (1970) J. Biol. Chem. 245, 6595-6598

Murray, K. N., Watson, J. G. \& Chaykin, S. (1966) J. Biol. Chem. 241, 4798-4801

Olson, J. S., Ballou, D., Palmer, G. \& Massey, V. (1974) J. Biol. Chem. 249, 4363-4382

Pick, F. M., McGartoll, M. A. \& Bray, R. C. (1971) Eur. J. Biochem. 18, 65-72

Stiefel, E. I. (1973) Proc. Natl. Acad. Sci. U.S.A. 70, 988-992

Tanner, S. J. \& Bray, R. C. (1978a) Biochem. Soc. Trans. 6, 230-232

Tanner, S. J. \& Bray, R. C. (1978b) Biochem. Soc. Trans. 6, 232-234

Tanner, S. J., Bray, R. C. \& Bergmann, F. (1978) Biochem. Soc. Trans. 6, 227-229

Tsopanakis, A. D., Tanner, S. J. \& Bray, R. C. (1978) Biochem. J. 175, 879-885 